\title{
Visual Topography of Human Intraparietal Sulcus
}

\author{
Jascha D. Swisher, ${ }^{1}$ Mark A. Halko, ${ }^{1}$ Lotfi B. Merabet, ${ }^{1,2}$ Stephanie A. McMains, ${ }^{1,3}$ and David C. Somers ${ }^{1,4}$ \\ ${ }^{1}$ Perceptual Neuroimaging Laboratory, Program in Neuroscience and Department of Psychology, Boston University, Boston, Massachusetts 02215, ${ }^{2}$ Center \\ for Noninvasive Brain Stimulation, Department of Neurology, Beth Israel Deaconess Medical Center, Harvard Medical School, Boston, Massachusetts \\ 02215, ${ }^{3}$ Neuroscience of Attention and Perception Laboratory, Department of Psychology, Princeton University, Princeton, New Jersey 08544, and \\ ${ }^{4}$ Martinos Center for Biomedical Imaging, Department of Radiology, Massachusetts General Hospital, Charlestown, Massachusetts 02129
}

Human parietal cortex is implicated in a wide variety of sensory and cognitive functions, yet its precise organization remains unclear. Visual field maps provide a potential structural basis for descriptions of functional organization. Here, we detail the topography of a series of five maps of the contralateral visual hemifield within human posterior parietal cortex. These maps are located along the medial bank of the intraparietal sulcus (IPS) and are revealed by direct visual stimulation during functional magnetic resonance imaging, allowing these parietal regions to be routinely and reliably identified simultaneously with occipital visual areas. Two of these maps (IPS3 and IPS4) are novel, whereas two others (IPS1 and IPS2) have previously been revealed only by higher-order cognitive tasks. Area V7, a previously identified visual map, is observed to lie within posterior IPS and to share a foveal representation with IPS1. These parietal maps are reliably observed across scan sessions; however, their precise topography varies between individuals. The multimodal organization of posterior IPS mirrors this variability in visual topography, with complementary tactile activations found immediately adjacent to the visual maps both medially and laterally. These visual maps may provide a practical framework in which to characterize the functional organization of human IPS.

Key words: retinotopy; parietal cortex; individual differences; vision; multisensory; tactile

\section{Introduction}

Posterior parietal cortex supports an extensive range of sensory and cognitive functions, including spatial representation, multimodal integration, attentional control, numerosity judgments, motor planning, and working memory (for review, see Kastner and Ungerleider, 2000; Culham and Kanwisher, 2001; Yantis and Serences, 2003; Behrmann et al., 2004; Grefkes and Fink, 2005; Hubbard et al., 2005; Merriam and Colby, 2005; Mesulam et al., 2005; Wagner et al., 2005; Buneo and Andersen, 2006; Orban et al., 2006). In macaque, a number of detailed cortical parcellation schemes (summarized in Van Essen, 2004) aid in the understanding of this functional diversity, but on an equivalent spatial scale, human parietal cortex remains poorly described.

In contrast, the organization of human occipital cortex and its relationship to that of nonhuman primates (Orban et al., 2004; Sereno and Tootell, 2005) is comparatively well understood. Occipital cortex is tiled with visual field maps that can be rapidly, precisely, and noninvasively identified using functional magnetic resonance imaging (fMRI) and phase-encoded stimuli (Engel et al., 1994; Sereno et al., 1995; DeYoe et al., 1996). These retinotopically defined borders provide important and practical "func-

Received Dec. 21, 2006; revised April 5, 2007; accepted April 5, 2007

This work was supported by National Science Foundation Grant BCS-0236737 (D.C.S.) and National Institutes of Health Grant K23 EY016131- 01 to (L.B.M.). Tactile patterns supplied by Steven Hsiao.

Correspondence should be addressed to Dr. Jascha D. Swisher, Program in Neuroscience, Boston University, 2 Cummington Street, Boston, MA 02215. E-mail: swisher@mind.bu.edu.

D0I:10.1523/JNEUROSCI.0991-07.2007

Copyright $\odot 2007$ Society for Neuroscience $\quad$ 0270-6474/07/275326-12\$15.00/0 tional landmarks" (Saxe et al., 2006) for the interpretation of task-related activations within individual subjects.

Equivalent approaches applied in parietal cortex have met with success. In the dorsal visual processing stream, conventional stimulus-driven retinotopic mapping has previously revealed a topographic structure extending anteriorly as far as area V7, defined as a map of the contralateral visual hemifield adjacent and superior to area V3A (Tootell et al., 1998; Press et al., 2001). Recently, several groups have used specialized stimuli to identify additional topographic representations in anterior (Sereno and Huang, 2006) and posterior (Sereno et al., 2001; Schluppeck et al., 2005; Silver et al., 2005) parietal cortex. The tasks used in these previous studies of posterior parietal cortex have relied on saccade planning or attention, without strong components of "bottom-up" sensory drive. These groups have identified one or two contiguous maps of the contralateral hemifield located dorsal to V7 on the medial bank of the posterior intraparietal sulcus (IPS). However, these parietal maps are less evidently well organized than the clearly defined topography of early visual cortex. The more recent reports (Schluppeck et al., 2005; Silver et al., 2005) find sporadic "dropouts" within the mapped regions where responses fall beneath statistical thresholds. Furthermore, the precise topography of these maps appears to vary substantially between individuals. It is unclear whether this apparent variability is attributable to limitations of the mapping technique, or whether it instead reflects true variability in the underlying topography of parietal cortex.

Using "checkerboard" retinotopic mapping stimuli and a conservative statistical approach, we find five hemifield maps, including V7, that extend along the medial bank of posterior IPS 
and into the superior parietal lobule. These maps are highly reproducible both in global structure and fine details across sessions within individual subjects and can often be identified without resorting to extensive signal averaging. Additionally, we find that intersubject variability in parietal visual topography is reflected in complementary patterns of activation produced in the same subjects by a simple tactile roughness judgment. Tactile responses are found both immediately adjacent to the visual maps and within the dropouts in the map complex. These precise, complementary activity patterns suggest that visual field maps may provide a useful and practical basis for descriptions of the multimodal functional organization of posterior parietal cortex.

Portions of this work have previously appeared in abstract form (Swisher et al., 2005).

\section{Materials and Methods}

\section{Subjects}

Twenty subjects were recruited from the Boston University community. All subjects provided informed consent and were scanned in accordance with MR safety guidelines under an experimental protocol approved by the Institutional Review Boards of Boston University and Massachusetts General Hospital. One author (M.A.H.) also served as a subject. Most subjects participated in two $1 \mathrm{~h}$ scan sessions, with high-resolution anatomical data collected in the first hour, followed by functional retinotopic mapping in the second session. Five subjects returned for additional intensive mapping sessions, as specified in Results. Three of these five also participated in separate sessions of delayed saccade mapping. Five subjects (three intensively mapped) additionally performed the tactile tasks in separate scan sessions.

\section{Stimulus-driven mapping protocol}

All visual stimuli were presented via a liquid crystal display projector illuminating a screen placed in the scanner bore. Subjects viewed the rear-projection screen through a mirror angled at $\sim 45^{\circ}$. The screen extended across a visual angle of $\sim 15^{\circ}$ radius horizontally and $12^{\circ}$ radius vertically.

The stimulus-driven mapping protocol resembled the well established procedures widely used to map the retinotopic organization of early visual areas (Engel et al., 1994; Sereno et al., 1995; DeYoe et al., 1996). The primary mapping stimulus was made up of a static background, viewed through a continuously moving transparent aperture in a dark foreground layer (supplemental Fig. 1, available at www.jneurosci.org as supplemental material). The background was a static, flickering chromatic radial checkerboard, with check radius scaled logarithmically in approximation of the cortical magnification function (Horton and Hoyt, 1991). Check luminance alternated spatially between "dark" and "light" checks, in addition to a random chromatic component, to provide both luminance and chromatic contrast. Dark and light checks reversed, and the chromaticity was randomly reassigned, at the $4 \mathrm{~Hz}$ flicker frequency.

The transparent aperture through which the checkerboard background was viewed comprised either a wedge rotating about fixation on polar angle mapping scans, or an expanding or contracting annulus on eccentricity mapping scans. The periodicity of both types of stimuli was $32 \mathrm{~s}$ ( 16 cycles per $512 \mathrm{~s}$ run). During the subsequent intensive mapping sessions, this periodicity was lengthened to $42.67 \mathrm{~s}$ ( 12 cycles $/ 512 \mathrm{~s}$ ). We alternated between clockwise and counterclockwise rotation (or expansion and contraction) on subsequent runs. The polar angle wedge was $90^{\circ}$ in width ( $72^{\circ}$ during intensive mapping) and extended to the edges of the screen, resulting in an asymmetry in the maximum peripheral extent of stimulation between the vertical and horizontal meridians. No substantive difference in response was observed between the two angular stimulus configurations used. During eccentricity mapping, the aperture increased logarithmically as a function of time in both size and rate of expansion, in an attempt to match the estimated human cortical magnification function and create a "traveling wave" of constant width and speed in early visual cortex. The duty cycle of the annulus was 5\% (any given point on screen was within the aperture for only $5 \%$ of the stimulus period). The ring expanded uniformly beyond the maximum extent of the screen and was offscreen entirely for $\sim 6 \mathrm{~s}$ of the $32 \mathrm{~s}$ stimulus period, after which it wrapped around to the fovea (vice versa for runs of contracting ring stimulation).

Subjects were instructed to fixate a small ( $\sim 8 \mathrm{arcmin})$ dot in the center of the screen and to respond with a button press whenever the fixation point dimmed. Dimming events occurred at random intervals throughout the scan, every $4.5 \mathrm{~s}$, on average. In a brief practice session before the scan, subjects viewed the stimulus and practiced the button press response while the importance of holding fixation was emphasized. During the scans, the subjects detected the dimming fixation point within $1 \mathrm{~s}$ on upward of $95 \%$ of trials. This task was not intended to be highly attentionally demanding, but rather simply to encourage subject vigilance and fixation during what would otherwise be an extraordinarily monotonous stimulus presentation.

\section{Delayed saccade mapping protocol}

The delayed saccade task was modeled after paradigms previously used by other investigators to investigate parietal topography (Sereno et al., 2001; Schluppeck et al., 2005). Subjects fixated a central point while a peripheral ( $\sim 11.4^{\circ}$ radius) target dot was briefly flashed $(250 \mathrm{~ms})$. The flashed peripheral stimulus was quickly replaced by a ring of 100 distractor dots randomly positioned within a ring of from 10.8 to $12^{\circ}$ radius. The distractors remained on screen for $3 \mathrm{~s}$, after which subjects made a saccadic eye movement to the remembered position of the peripheral target and then immediately made a saccade back to the central fixation point.

The position of the peripheral saccade target rotated around the periphery from trial to trial, so that after 12 trials the target completed one full cycle. A trial lasted $4.27 \mathrm{~s}$, with 10 cycles completed in a single $512 \mathrm{~s}$ scan.

\section{Tactile roughness judgment}

The stimulus and task used have been described in detail previously (Connor et al., 1990; Merabet et al., 2004, 2007). Briefly, raised dot patterns at one of seven dot densities were placed beneath the pad of the right index finger of blindfolded subjects. The dots were $1 \mathrm{~mm}$ in diameter, 2 $\mathrm{mm}$ in elevation, and arranged in tetragonal arrays, the interdot spacing of which varied from 1 to $7 \mathrm{~mm}$. Subjects' hands were positioned palm down by their side as they lay supine in the scanner. They made two scanning sweeps of the right index finger, judged the perceived roughness of the dot pattern on a 1-4 scale, and responded with a button press in the left hand. In a motor control condition, subjects made similar sweeping motions over a smooth surface and responded with random button presses. The tasks were presented in the scanner in $30 \mathrm{~s}$ blocks preceded by an auditory task cue. Each trial within the block lasted $\sim 5-7 \mathrm{~s}$.

\section{Scan parameters}

Data acquisition took place in either 3T Siemens (New York, NY) Allegra or 3T Siemens Tim Trio scanners located at the Martinos Center for Biomedical Imaging at Massachusetts General Hospital. fMRI scans used echo planar imaging (EPI) sequences sensitive to blood oxygenation level-dependent contrast [repetition time (TR), $2 \mathrm{~s}$; echo time (TE), 30 ms; voxels: $2.8 \times 2.8 \times 3.3 \mathrm{~mm}$ (Allegra), $3.125 \times 3.125 \times 3.0 \mathrm{~mm}$ (Trio)]. A custom-made surface coil (Advanced NMR, Wilmington, MA) was used with the Allegra and placed at or slightly dorsal to the occipital pole to maximize coverage of early visual and parietal areas. A 12-channel head matrix coil was used with the Trio. Only data from the initial scan sessions in the Allegra were used in the comparison of signal change across visual areas, described below. From 27 to 32 slices were oriented approximately perpendicular to the calcarine sulcus, in most subjects giving coverage of the brain as far anterior as the central sulcus. Eight seconds of data were discarded at the beginning of each functional scan.

Before functional imaging in each session, T1-weighted EPI images were acquired using the same slice prescription as the functional scans to allow for registration of the data from each session to a subject's highresolution anatomical images. The high-resolution anatomical volume was imaged in a previous session for each subject using an MPRAGE (magnetization-prepared rapid gradient echo) sequence with $1 \times 1 \times$ $1.33 \mathrm{~mm}$ resolution. 
Statistics and analysis

Data preprocessing. Functional data were motion corrected (Cox and Hyde, 1997) and intensity normalized before manual registration to an individual subject's high-resolution anatomical volume.

Retinotopy analysis. Phase-encoded retinotopic mapping (Engel et al., 1994; Sereno et al., 1995; DeYoe et al., 1996) is based on the use of a periodic stimulus to induce neural activity at the same frequency in visually responsive voxels. Two components of the response are of interest: the amplitude of the response at the stimulus frequency relative to the background spectrum determines the significance of the modulation, whereas the phase of this response encodes the spatial position of the receptive field of the voxel.

Multitaper spectral estimation. We used Thomson's multitaper method to generate a smoothed estimate of the spectrum of the time course at each voxel (Thomson, 1982; Percival and Walden, 1993; Mitra and Pesaran, 1999). The multitaper method is a nonparametric form of spectral analysis in which time series data are first windowed by a set of $\mathrm{K}$ mutually orthogonal tapers, thereby producing K-independent spectral estimates that have been smoothed in the frequency domain. The particular tapers used are from the family of discrete prolate spheroidal sequences (Slepian, 1978), which are finite, orthonormal sequences, the energy of which is maximally concentrated within a user-defined bandwidth. This bandwidth is often expressed implicitly as the time-bandwidth product $\mathrm{NW}$, where $\mathrm{N}$ is the sequence length (e.g., in seconds) and $\mathrm{W}$ is the design bandwidth (e.g., in hertz). Typical values for NW are in the range 2-4, with greater values indicating a greater degree of smoothing: the number of Rayleigh frequencies over which the spectral estimate is smoothed is given by $2 \mathrm{NW}$. NW was set to 4 in this study.

Multitaper test for periodicity. The statistical methods previously used in retinotopic mapping [including those based on coherence measures or global $F$ statistics (for description, see Tootell et al., 1998)] require the assumption of a strictly white background spectrum, an assumption that is known not to hold for fMRI noise (Zarahn et al., 1997). A statistical test associated with the multitaper estimator (Thomson, 1982) detects sinusoidal components that are significantly greater in amplitude than a slowly changing background spectrum. This test requires only the assumption of "locally white" noise, where "local" refers to the smoothing diameter $2 \mathrm{NW}$. The test produces an $F$ ratio with 2 and $2 \mathrm{~K}-2$ degrees of freedom, where the numerator estimates the amplitude of a potential harmonic component at the specified frequency and the denominator estimates the local background spectrum, assumed to be noise. Here, $\mathrm{K}$ is set to equal $2 \mathrm{NW}-1$, thereby including only those tapers with potentially good bias properties (Percival and Walden, 1993). The multitaper test requires that the background spectrum be slowly changing in the vicinity of the signal, which is an assumption easily met at moderate to fast stimulus frequencies. Thus, in contrast to previous tests, the significance values determined by the multitaper test are valid for statistical inference, which is of critical importance when attempting to efficiently detect weak signals. This approach has been previously applied to brain imaging data (Mitra and Pesaran, 1999) but has not, to our knowledge, been used previously for the detection of retinotopic maps. In practice, the multitaper test has slightly less statistical power than tests previously used in retinotopic mapping (e.g., global $F$-statistic-based tests), because of a reduction in the degrees of freedom of the background noise amplitude estimate, but in return produces significance values that remain valid even in the presence of substantial low-frequency noise (for more details, see supplemental Figs. 2, 3, available at www.jneurosci.org as supplemental material).

Thomson's multitaper test was performed at the known stimulation frequency for each voxel in each run. Because each run can be considered to be independent, the voxelwise $F$-statistic numerator and denominator of each run were summed separately to produce a combined $F$ statistic for the scan session with $2 \times$ Nruns and $(4 \mathrm{NW}-4) \times$ Nruns degrees of freedom.

Response phase estimation. The sinusoidal component at the stimulus frequency is estimated by complex-valued regression as part of the multitaper test for significance. For significantly active voxels, the phase of this estimated sinusoid is nearly identical to that obtained by the usual method of calculating the Fourier transform of the time series directly.
The phase estimate was combined across clockwise and counterclockwise (expanding/contracting) runs by taking the complex conjugate of the signal estimates in the counterclockwise (contracting) runs, thereby reflecting the phase about 0 (Sereno et al., 1995), and summing the resulting estimates across all runs via simple vector addition and reporting the phase of the resulting vector sum.

\section{Visualization on cortical surface}

The above analysis produces four summary volumes ( $F$-statistic numerator, $F$-statistic denominator, real and imaginary components of phase estimate) for each mapping stimulus (ring or wedge) per session. These volumes were upsampled by a factor of 4 in each dimension using linear interpolation to reduce aliasing artifacts in the projection onto the higher-resolution anatomical volume. No explicit smoothing was applied to the functional data at any stage of the analysis.

The cortical surface of each hemisphere of each subject was reconstructed as a triangular mesh from the subject's automatically segmented high-resolution anatomical volume using Freesurfer software (Dale et al., 1999; Fischl et al., 1999). Both the gray/white matter and pial surfaces were segmented. The upsampled functional statistic volumes were projected by nearest neighbor search onto the vertices of an interpolated mesh located halfway between the gray/white and pial surfaces. The cortical mesh was then computationally inflated.

Flattened representations of parietal cortex were generated by first separating the superior parietal surface from the remainder of the inflated cortex with a linear cutting plane. The resulting semicylindrical surface of each hemisphere of each subject was then computationally flattened with Freesurfer software so as to minimize both angular and distance distortion (Fischl et al., 1999). For display purposes, the flattened surface patches were cropped into a rectangle with the posterior branch of the IPS aligned along the long axis. In some hemispheres, the edge of the cutting plane falls partly within the cropped patch, appearing as a ragged border within the rectangular boundaries of the cropped image.

The projected functional statistics were used to combine mapping data across sessions on separate days. The significance of the periodic activation across sessions was assessed by separately summing across sessions the projected $F$-statistic numerators and denominators at each vertex on the cortical mesh and taking their ratio to produce a single cross-sessioncombined $F$ statistic per surface vertex. These $F$ statistics were converted to significance values and used to threshold the visualization of the maps, as described below. We generated cross-session phase estimates by first weighting the real and imaginary phase components of each session by the within-session $F$ ratio, multiplied by the number of runs in the session. This weighting is proportional to the square of the within-session signal-to-noise ratio (SNR), which is the least-squares optimal weighting for a combined phase estimate at moderate to high SNR (Warnking et al., 2002). The resulting weighted real and imaginary components were then summed vertexwise over the cortical surface, and the final vector sum at each vertex was converted back into an angular phase measurement.

The resulting projected statistical image was then visualized similarly to previous reports (Sereno et al., 1995). Briefly, surface vertices, the significance of which exceeded a specified threshold, were rendered in a color indicating the phase of the signal estimate at that point. The color map was defined so that the representation of the contralateral horizontal meridian was rendered in blue, the upper vertical meridian was rendered in red, the lower vertical meridian was rendered in green, and the ipsilateral horizontal meridian was rendered in yellow. For display purposes, the color map was compressed slightly to overrepresent the contralateral visual field, so as to approximately match the distribution of response phase within the early retinotopic areas as described in Results. Surface vertices, the significance of which did not exceed threshold, were rendered in dark or light gray to reveal cortical sulci and gyri. All maps shown here are thresholded at a $p<0.05$ vertexwise level, except where otherwise indicated.

\section{Region-of-interest definitions}

Two sets of regions of interest (ROIs) were defined for each hemisphere of each subject on their reconstructed cortical mesh. The first set in- 

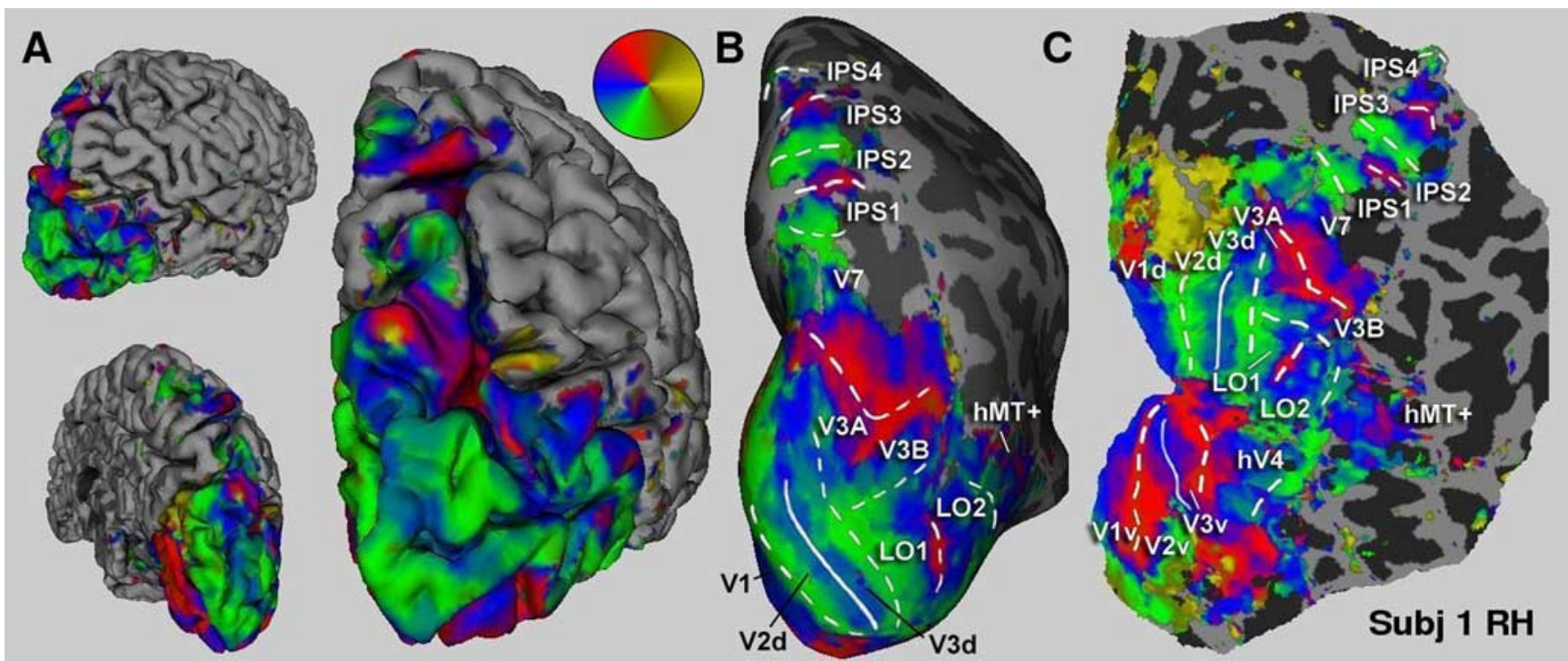

Figure 1. The angular mapping wedge stimulus reveals visuotopically specific activation throughout a substantial portion of the posterior cerebral cortex. The angular position of the wedge resulting in the greatest significant $(p<0.05)$ response is indicated by the colored overlay (inset), with red representing the upper visual meridian, blue representing the contralateral horizontal meridian, and green representing the lower meridian. Potential ipsilateral responses are indicated in yellow. The right hemisphere (RH) of a single subject (Subj 1 ) is shown here. $A$, The reconstructed pial surface is shown from posterior lateral (top left), posterior medial (bottom left), and posterior (right) views. B, Computational inflation of the folded cortical surface reveals visuotopically specific activation within the sulci. Reversals in the represented angular position correspond to areal boundaries in the early visual areas and are shown as overlaid lines. Dashed lines indicate reversals at representations of the vertical meridian, whereas solid lines indicate reversals at horizontal meridian representations. We find five hemifield maps along the medial bank of the IPS, including area V7 and IPS1-IPS4. C, A flattened representation of the occipital cortex (extending into parietal and temporal regions) showing 14 simultaneously mapped visual regions. The disorganized responses in the peripheral regions of V1-V3 likely represent negative blood oxygenation level-dependent signal, leading to lagged or antiphase responses to the visual stimulus (Shmuel et al., 2002).

cluded ROIs defined primarily on retinotopic criteria, intended to correspond to the underlying visual maps. ROIs were defined for each quadrant of early retinotopic visual cortex (V1-V3) and for the V3A and V7 hemifield representations. In parietal cortex, ROIs were defined by the posterior and anterior phase reversals of each hemifield map in the angular dimension (typically anterior/posterior) and the limits of significant $(p<0.05)$ angular response in the orthogonal (typically medial/ lateral) dimension. In those subjects where activation was not sufficient to allow map boundaries to be determined based on functional criteria alone, we defined similar V7 and IPS1-4 ROIs by reference to the sulcal anatomy. All of these parietal regions were defined to be approximately rectangular and contiguous with their neighboring ROIs. These ROIs were used in the comparison of signal change across visual maps and for the calculation of cross-session phase correlation, described below.

The second group of ROIs were large, anatomically defined regions centered on the posterior IPS. The borders of these regions closely followed the boundaries of the cropped parietal surface patches shown in Figures 4 and $6-8$. These ROIs were used to define the region included in the calculation of correlations between percentage signal change images, described below.

\section{Test-retest reliability}

We assessed the test-retest reliability of the visuotopic maps in terms of both their amplitude and their phase. Reproducible amplitudes of activation show that the activated regions of cortex are similar from one session to the next, whereas reproducible phases demonstrate that the maps within these areas remain aligned between sessions. The reproducibility of the activation patterns was assessed by first normalizing the functional statistical volumes into percentage signal change units and then upsampling and projecting these volumes onto the reconstructed cortical surface as described previously. We then calculated the correlation between percentage signal change images from each session (Haxby et al., 2001) within a large anatomically defined posterior parietal ROI. The mean signal change within the ROI was subtracted from the activation images separately for each session before the correlation being computed. High correlations indicate good reproducibility of the activation patterns between mapping sessions. The same method was also used to evaluate the relationship between the patterns of visuotopic and tactile activation. A negative correlation indicates complementary patterns, with high tactile drive in regions of low visuotopic activation and vice versa.

The reproducibility of the phase maps within the visuotopically activated cortex was assessed separately for each cortical map by calculating the circular correlation (Fisher and Lee, 1983) between the phase estimates of points within the ROI that were significantly $(p<0.05)$ modulated in both sessions. We evaluated the significance of these correlations via a permutation test. At each iteration of this test, the phases of the set of significant voxels intersecting the ROI were randomly permuted and projected onto the cortical surface (without upsampling), after which the circular correlation between significant vertices was calculated. This was repeated 10,000 times for each ROI per subject, creating an estimate of the distribution of correlation values under the null hypothesis. The significance of the original correlation was then determined with respect to this simulated null distribution.

\section{Tactile data analysis}

Analysis of the tactile session data used standard procedures and software (FS-FAST, the FreeSurfer Functional Analysis STream; CorTechs, La Jolla, CA). Briefly, the voxelwise time series were fit by a general linear model, the regressors of which matched the time course of the experimental conditions as smoothed by a canonical hemodynamic response function. The contrast of the roughness judgment versus the motor control conditions produced a volume of $t$ statistics for each subject, which were then converted into significance values and upsampled and projected onto the subject's reconstructed cortical surface as described previously for the retinotopy data.

\section{Results}

We used fMRI to map the representations of the visual field found within human occipital and parietal cortex. Previous studies using similar methods have identified the hemifield maps V3A, V3B, V7, LO1, and LO2 (Larsson et al., 2006) on the dorsal and lateral aspects of the human occipital lobe, in addition to the well known early visual areas V1, V2, and V3. Here, we simultaneously map an additional four representations of the contralat- 
Table 1. MNI coordinates (in millimeters)

\begin{tabular}{lllllrr}
\hline & V3A & V3B & V7 & IPS1 & IPS2 & IPS3 \\
\hline Mean & $\pm 20,-90,23$ & $\pm 33,-85,16$ & $\pm 26,-79,31$ & $\pm 23,-73,40$ & $\pm 21,-68,52$ & $\pm 25,-61,55$ \\
SD & $6,4,6$ & $6,7,7$ & $6,6,7$ & $6,7,7$ & $\pm, 7,8$ & $7,7,6$ \\
\hline
\end{tabular}

MNI, Montreal Neurological Institute.

eral visual hemifield extending along the medial bank of the IPS (Fig. 1, Table 1).

Areas V3A and V3B lie along the transverse occipital sulcus (Tootell et al., 1997; Smith et al., 1998; Press et al., 2001). Anterior and adjacent to area V3A, along the inferior portion of the medial bank of the IPS, is the V7 hemifield representation (Tootell et al., 1998; Press et al., 2001) (Fig. 2 ). We find reliable, topographically specific responses to direct visual stimulation well beyond this point, extending superiorly along the medial bank of the posterior IPS and further into the superior parietal lobule. Within this region, the representation of angular position in the visual field changes continuously in the direction parallel to the length of the sulcus, with reversals at the representations of the upper and lower visual meridians. The first two hemifield representations beyond V7 are consistent in topography and anatomical location with the recently described IPS1 and IPS2 maps, which previously have been revealed only by more cognitive, "top-down" tasks such as delayed saccades (Sereno et al., 2001; Schluppeck et al., 2005) or covert attention (Silver et al.,

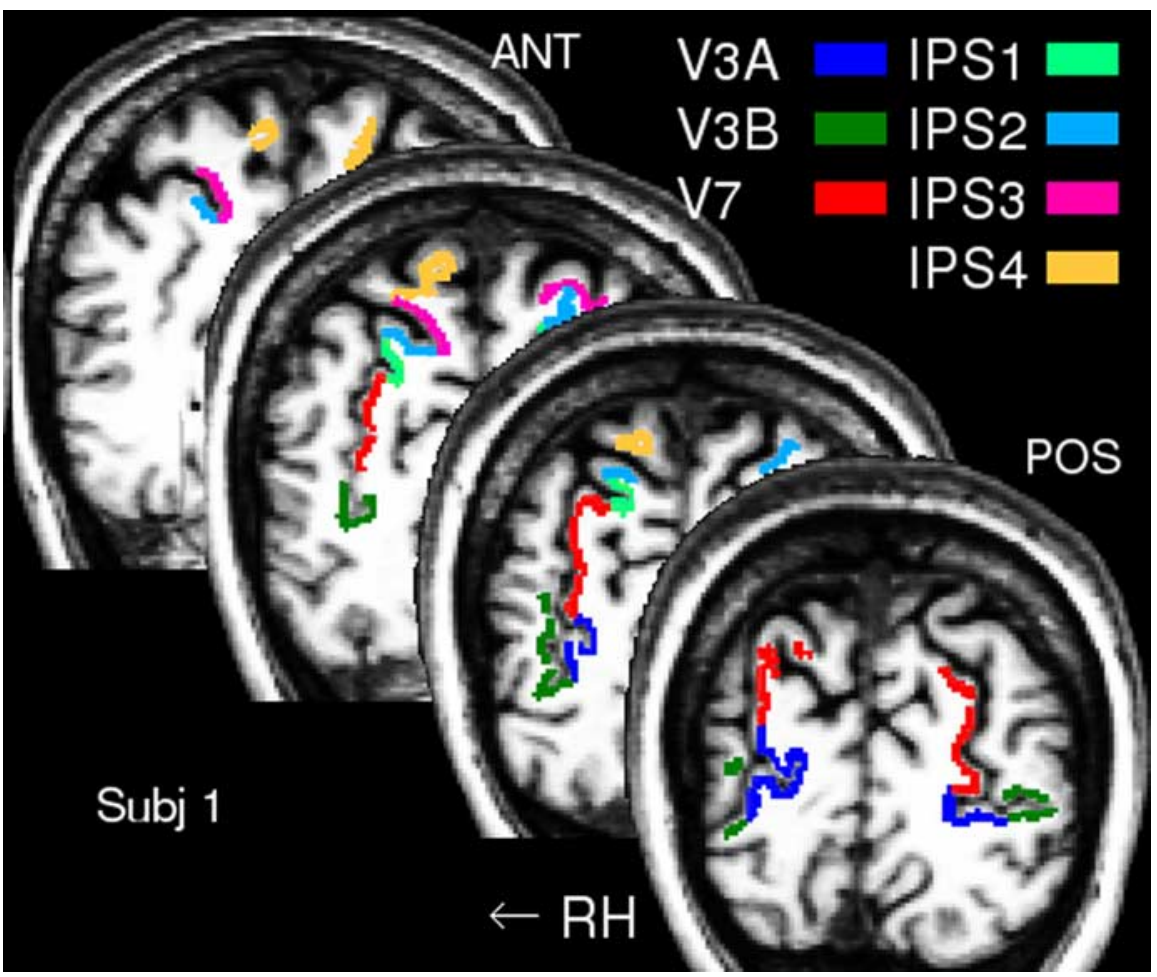

Figure 2. Coronal slices. The location of V3A, V3B, V7, and IPS1- 4 is indicated in coronal slices through the brain of subject 1 (Subj 1). Area V7 occupies a substantial portion of posterior/inferior IPS. RH, Right hemisphere; ANT, anterior; POS, posterior.
2005). In many subjects, one to two addi-

tional hemifield maps are found anterior to IPS2, usually continuing to parallel the sulcal anatomy as it turns mediolaterally. In keeping with the naming convention established for the more posterior parietal maps, we refer to these additional two hemifield representations as IPS3 and IPS4.

These parietal regions responded predominantly to stimuli presented in the contralateral visual field (Fig. 3). As reported in the covert attention studies of Silver et al. (2005), the vertical meridian is underrepresented, with the contralateral hemifield representation appearing to be compressed toward the horizontal meridian. We also find a similar, although smaller, horizontal bias in early visual cortex. A horizontal overrepresentation has also been reported using fMRI in the human lateral geniculate nucleus (Schneider et al., 2004).

\section{Eccentricity organization}

Standard thin-ring stimuli (Tootell et al., 1997) revealed a representation of the eccentricity dimension of the visual field approximately orthogonal to the angular dimension on the cortical surface, although these responses were somewhat less robust than those to the angular mapping wedge stimuli (Fig. 4). V3A and $\mathrm{V} 3 \mathrm{~B}$ are separated by a local minimum in the eccentricity map; however, as reported previously (Press et al., 2001), this minimum often does not appear to represent the central $3^{\circ}$ of the visual field. We reliably find a continuous gradient of eccentricity response phase along the V7/IPS1 border. This gradient reveals a mediolateral eccentricity representation, with a laterally posi- tioned foveal representation in the fundus of the IPS, moving through parafoveal representations on the medial sulcal wall, to representations of the peripheral extent of stimulation $\left(12-15^{\circ}\right.$ radius) on the adjacent gyrus. This lateral central field representation appears to be the same as that reported previously within area V7 (Press et al., 2001; Tyler et al., 2005; Wandell et al., 2005); however, the present angular phase maps indicate that it is more properly considered to belong to both the V7 and IPS1 hemifield maps, defining a map complex or cluster by virtue of the confluent foveal representation (Balasubramanian et al., 2002; Wandell et al., 2005). Although the representation of eccentricity within IPS3/4 is less clear, a parsimonious interpretation of the data suggests that these anterior regions continue the pattern of foveal responses laterally and peripheral responses medially seen in the more posterior IPS, likely forming a second distinct foveal representation.

\section{Signal change across areas}

To compare the strength of parietal responses to those in earlier visual areas, we calculated the voxelwise percentage signal change at the fundamental frequency of the angular mapping stimulus. Each subject's volume of voxelwise angular mapping percentage signal change estimates was interpolated and projected onto the cortical surface, and the percentage signal change for each point within an ROI was averaged together. A second "null" signal change was also calculated for each area, defined as the estimated signal change at a frequency five cycles per run greater than the 

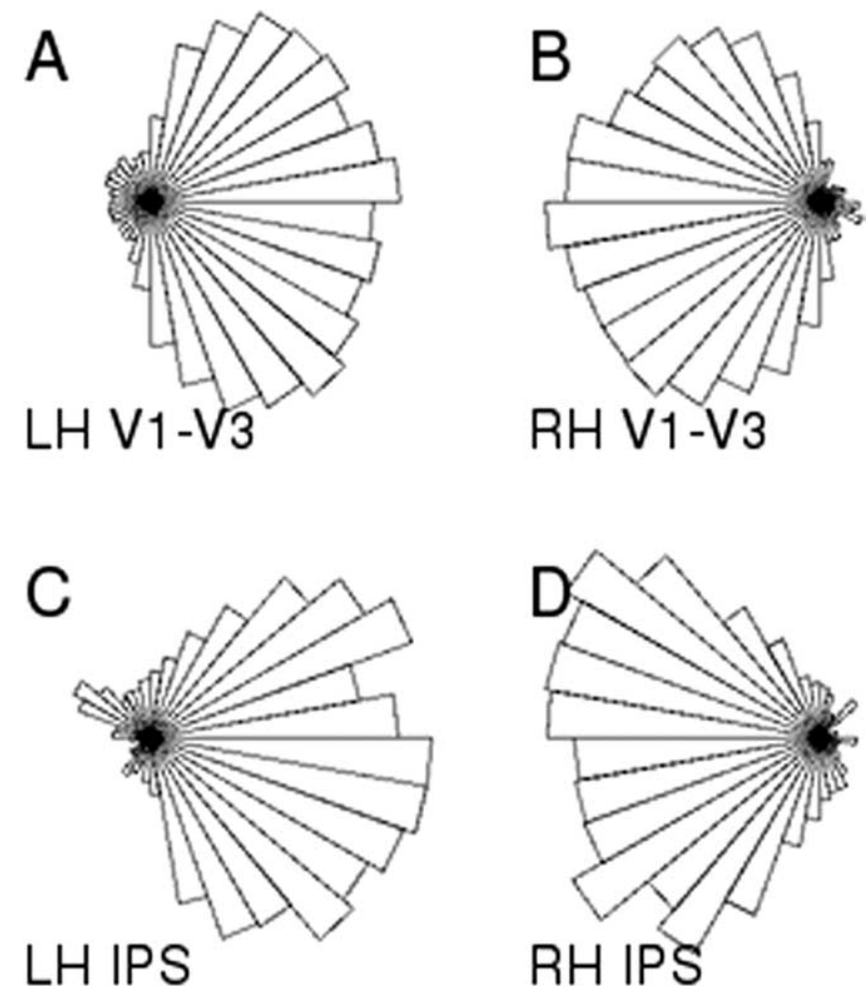

Figure 3. Radial histograms of preferred angular response phase. These rose diagrams show the distribution of response phase for surface vertices responding significantly to the angular mapping stimulus, combined across all five intensively mapped subjects. The histograms have been rotated so that the angular position on the plot corresponds to the angular position within the visual field. $\boldsymbol{A}, \boldsymbol{B}$, Histograms for left and right combined V1, V2, and V3. C, D, Histograms for left and right combined V7 and IPS1-4. All respond predominately to contralateral stimuli. Both early visual and parietal cortex overrepresent the horizontal meridian, although the overrepresentation is somewhat more pronounced in IPS. The color maps used to display the response to the angular mapping stimulus (as in Fig. 1) are defined to similarly overrepresent the contralateral hemisphere. LH, Left hemisphere; RH, Right hemisphere.

stimulus frequency. No periodic response is expected at this null frequency.

As expected, periodic visual responses in parietal cortex are much smaller than in early visual areas (Fig. 5A). Response amplitude drops off substantially between V3A and V7 and decreases gradually as one moves into higher parietal areas, reflecting, in part, increased distance from the surface coil. Across the full population of 20 subjects scanned, signal change is significantly greater at the actual stimulus frequency than at the null frequency within all areas up to and including IPS4 (Wilcoxon rank test, all $p<10^{-5}$ ). These responses are sufficiently strong as to often allow the identification of these visual maps without resorting to extensive signal averaging. Significant $(p<0.05$ voxelwise) responses were observed in IPS1/2 in 14 of 20 subjects and in IPS3/4 in 11 of 20 subjects, with 512 time points of data acquired ( $\sim 18$ min of scanning with a TR of $2.0 \mathrm{~s}$ ).

\section{Test-retest reliability}

Five subjects with relatively clear parietal maps revealed during the initial scan sessions were brought back for subsequent intensive mapping, allowing the test-retest reliability of these measurements to be examined. The intensive sessions took place between 2 and 18 months after initial mapping, over which time the parietal maps proved to be highly stable (Fig. 6). We examined the reliability of these measurements both in terms of the cortical regions activated and of the visual field maps revealed within these activated areas. Across the population of five repeatedly scanned subjects ( 10 hemispheres), the activation patterns within posterior parietal cortex (as measured by percentage signal change, thereby disregarding response phase) were highly correlated between sessions, with a mean correlation of $r=0.77$ across 10 hemispheres (median, $r=0.79$; maximum, 0.86 ; minimum, 0.68 ), demonstrating that the mapping stimulus reliably activated the same areas of cortex across sessions.

Within each defined parietal map, the response phases were also well correlated between sessions. We initially determined a cross-session phase correlation for each ROI in each hemisphere by calculating the circular correlation (Fisher and Lee, 1983) across sessions of the response phases of all points within the ROI that were significantly $(p<0.05)$ active in both sessions. We then averaged these values across all hemispheres to find a mean population correlation for each ROI (Fig. 5B). There was one outlier (subject 5, left hemisphere) who produced a spurious negative correlation $(r=-0.66)$ in IPS2, based on five active voxels. This hemisphere had zero overlapping voxels between sessions in IPS3 and IPS4. Accordingly, the hemisphere was excluded in the calculation of correlations for IPS $/ 3 / 4$. The resulting population correlation was significantly greater than expected by chance (Wilcoxon rank test) within all parietal ROIs.

For a more stringent test of the reproducibility of these maps within individual subjects, we contrasted the observed test-retest circular correlation within each ROI versus correlations obtained from random permutations of the maps. This tests whether the between-sessions correlation for the ROI of a particular hemisphere is greater than would be expected from a random sampling of voxels of similar spatial extent and with similar visual field preferences. The test is sensitive both to the similarity in response phase of matching points as well as the amount of spatial overlap between sessions, with greater overlap producing a more significant correlation for equal agreement in phase at individual points. Each identified parietal map was found to be significantly $(p<0.05)$ reproducible by this strict criterion in at least half of the mapped hemispheres, with 8 of 10 hemispheres showing significant correlations in V7 and IPS1, 5 of 10 showing significant correlations in IPS2, 6 of 10 showing significant correlations in IPS3, and 5 of 10 showing significant correlations in IPS4. Maps that were not found to be significantly correlated across sessions were almost uniformly within small or minimally active ROIs, and thus these failures to reach significance were likely attributable to limited statistical power, rather than to gross misalignment of the phase maps between sessions.

\section{Comparison with delayed saccades}

Previous reports of visual topography in human parietal cortex have often used a delayed saccade task (Sereno et al., 2001; Schluppeck et al., 2005). To compare the topography revealed by these differing paradigms, three subjects with clear, continuous map structures were rescanned while performing a delayed saccade task similar to those used previously by other groups. We find the pattern of activation during delayed saccade mapping is typically more sparse than that generated by stimulus-driven mapping (given equal acquisition times), but that both tasks activate the same regions of cortex (Fig. 7). The overall topography is in reasonable agreement between the tasks, with an average correlation between saccade and stimulus-driven percentage signal change data of $r=0.36$ (median, $r=0.40$; maximum, 0.43; minimum, $0.18 ; n=6$ hemispheres). Across this small sample, we found significant phase correlations $(p<0.05$, Wilcoxon rank test) between saccade and stimulus-driven maps within 


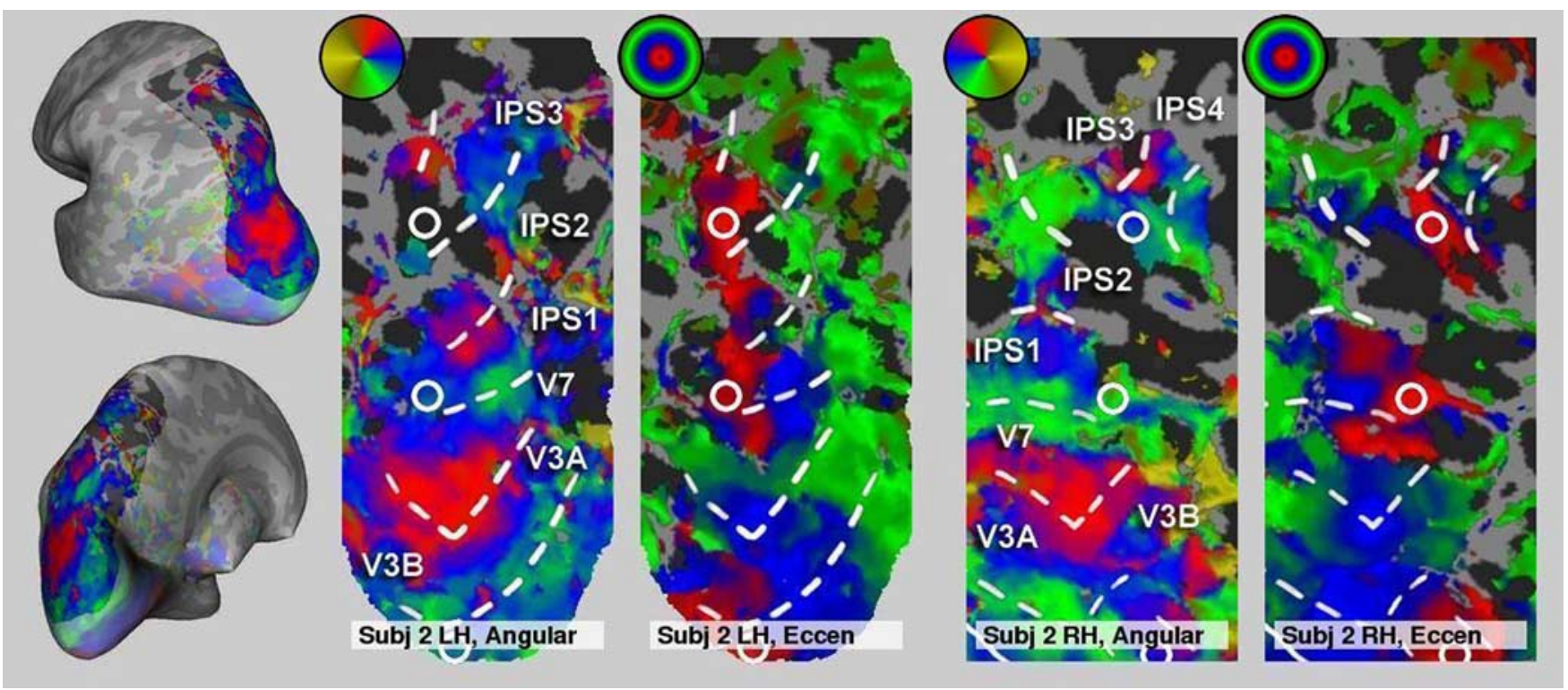

Figure 4. Angular and eccentricity patches for the left (LH) and right (RH) hemispheres of a second subject (Subj 2). The location of the patches is indicated by shading on the inflated hemisphere (far left). The patches are aligned so that posterior IPS runs vertically, from V3A/B at the bottom (inferiorly) to IPS4 at the top (superiorly). Angular (left) and eccentricity (Eccen; right) data are displayed on the same anatomical patches, with identical borders overlaid. The eccentricity mapping color key goes from red at central eccentricities (here $\sim 0-3.5^{\circ}$ radius) through mideccentricities in blue and the periphery in green. Foveal responses are indicated by circles.

V7 and IPS1 and a trend toward significant correlation in IPS2 $(p<0.1)$.

\section{Individual differences and multisensory activation}

Although the large-scale pattern of multiple alternating hemifield maps along the IPS is evident in nearly all subjects, individuals exhibit substantial variability in the fine details of this topography. To a large degree, this variation in functional organization appears to reflect individual differences in the anatomy of the IPS and surrounding gyri, which can vary greatly even between hemispheres of the same subject (Ono et al., 1990). In particular, the IPS3/4 maps are often, but not always, found to be rotated mediolaterally relative to IPS1/2, usually following the local anatomy of the sulcus at that point.

Many individuals show an apparent dropout in the map complex within IPS1 or IPS2 (Fig. 7) (Schluppeck et al., 2005; Silver et al., 2005). These dropouts are reproducible features of the activation patterns, suggesting that they do not simply reflect random deviations below statistical threshold as a result of insufficient signal averaging. Because IPS is generally regarded as an area of multimodal integration (Grefkes and Fink, 2005), one specific alternative hypothesis is that these dropouts may be responsive to stimulation in other sensory modalities. In particular, tactual roughness discriminations have been shown to activate posterior parietal areas, potentially overlapping these visuotopically organized regions (Kitada et al., 2005, 2006; Merabet et al., 2007). To test this, we compared the location of these parietal visual maps to the activation patterns produced in five blindfolded subjects as they performed a simple tactile roughness judgment with the index finger of the right hand. The two patterns were strikingly complementary, with significant tactile activations immediately adjacent to the significant visuotopic responses on both medial and lateral sides. There was often slight overlap at the boundaries of the two regions, but even at a lenient statistical threshold ( $p<$ $0.1)$ the areas responsive to visual and tactile modalities were never observed to fully overlap (Fig. 8 and supplemental Fig. 5, available at www.jneurosci.org as supplemental material). Cru- cially, however, in every hemisphere where an extensive dropout in the visuotopic maps at the level of IPS1/2 resulted in two discontinuous regions of significant $(p<0.05)$ parietal visuotopic activation, we observed significant $(p<0.05)$ tactile activation spanning the visuotopic dropout. This significant tactile activation typically connected two stronger activation foci medial and lateral to IPS, resulting in a single contiguous region of tactile activity extending across the sulcus. Conversely, in hemispheres where significant visuotopic activation extended in a single contiguous band parallel to the sulcus, tactile activation was always separated by the visuotopic complex into two distinct foci medial and lateral to the sulcus. These qualitatively complementary patterns are reflected quantitatively in an anticorrelation between the parietal percentage signal change patterns for the two modalities (mean, $r=-0.28$; maximum, -0.16 ; minimum, $-0.42 ; 10$ hemispheres). ROIs for this comparison were defined based on anatomic criteria and corresponded to the regions of cortex shown in the patches of Figure 8.

\section{Discussion}

Detailed retinotopic mapping permitted the simultaneous identification of up to 14 distinct visual field maps in the occipital and parietal lobes of human subjects, including five contiguous regions extending along the medial bank of IPS and into the superior parietal lobule. Two of these IPS areas, IPS3 and IPS4, are newly defined. Two of the IPS areas, IPS1 and IPS2, are reported here using direct stimulus mapping; previous reports have used higher-order saccade or spatial attention mapping tasks (Sereno et al., 2001; Schluppeck et al., 2005; Silver et al., 2005). The most posterior region, V7, which has previously been reported via retinotopic mapping (Tootell et al., 1998; Press et al., 2001; Tyler et al., 2005), is shown here to lie in IPS and to share a fovea representation with IPS1. These stimulus-driven visual field maps provide an important functional parcellation of IPS, which should help guide future investigations of the mechanisms of spatial attention, multisensory integration, visuospatial motor planning, 


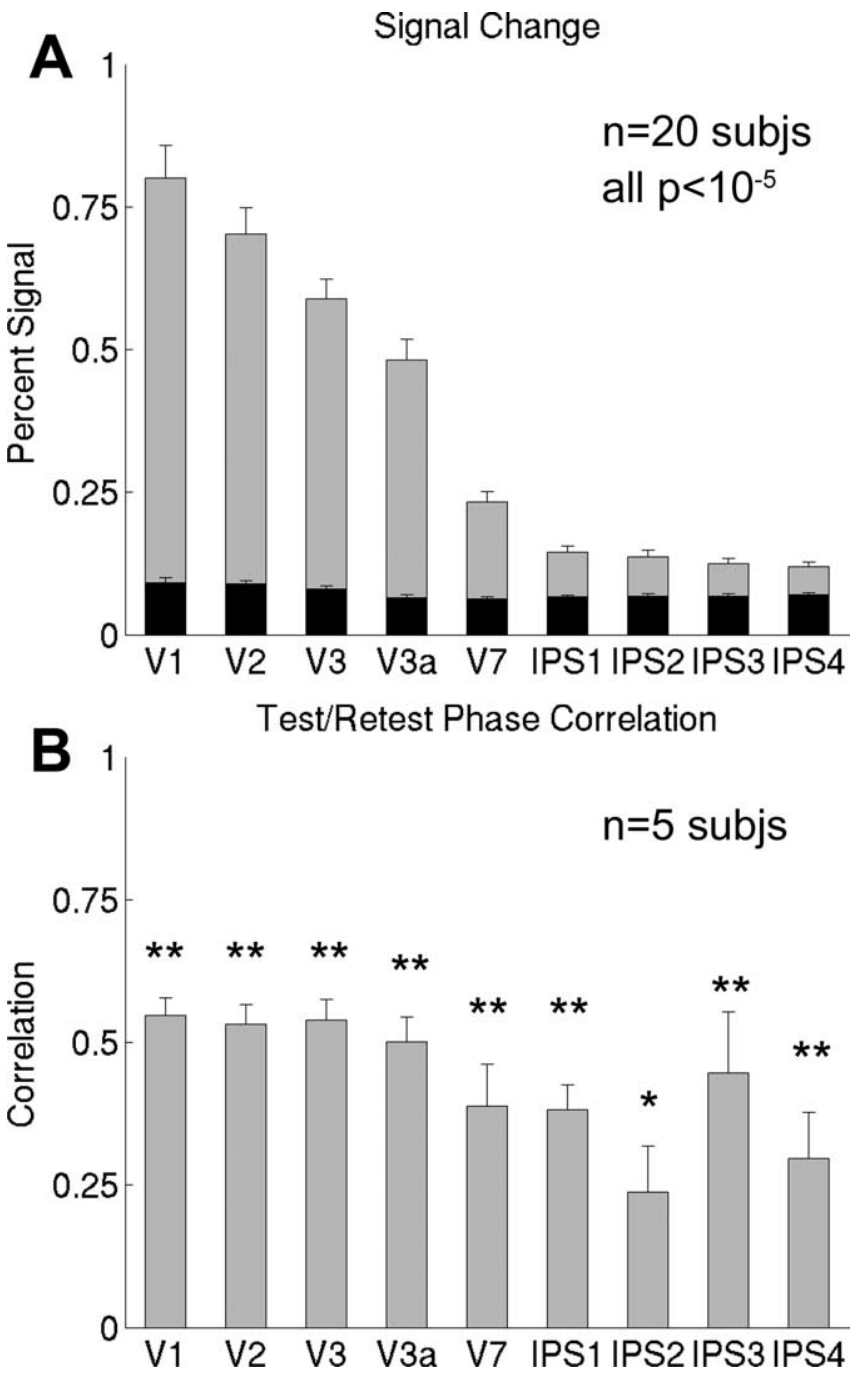

Figure 5. Average amplitude and reproducibility of visuotopic activity across areas. $\ln \boldsymbol{A}$, gray bars show the average signal change (mean to peak) within the retinotopically defined visual maps at the mapping stimulus frequency across the full population of 20 subjects (subjs) scanned. Black bars show the signal change estimate at a frequency five cycles per run greater than the mapping stimulus, at which no significant periodic component is expected. In $\boldsymbol{B}$, bars show the circular correlation between the phases of points significantly active in both mapping sessions over the set of five repeatedly scanned subjects. Error bars indicate SEM. ${ }^{*} p<0.05$; ${ }^{* *} p<0.01$

and the other cognitive functions that have been shown to use intraparietal cortical circuitry.

These visual field maps were detected using multitaper analysis, a statistical approach that remains valid even under conditions of low-frequency noise (Thomson, 1982; Zarahn et al., 1997), leading to more conservative estimates of the significance of the activations than in previous phase-encoded mapping studies. We further note that variability between subjects in the precise pattern of visuotopic response is reflected in complementary activation patterns produced in parietal cortex during a simple tactile roughness judgment performed while blindfolded. This suggests that these apparent intersubject differences in parietal topography do reflect true underlying variability in cortical organization, rather than artifactual or methodological limitations.

\section{Comparison with previous mapping}

Previous investigations using stimulus-based mapping paradigms have not consistently detected these parietal hemifield maps, leading to the belief that explicit covert attention or other cognitive factors may be critical to revealing parietal topography. As a result, visual mapping studies targeting the parietal lobes have generally used more cognitively demanding tasks, including covert attention tasks (Silver et al., 2005) and delayed saccades (Sereno et al., 2001; Schluppeck et al., 2005). The use of unique stimuli for each region to be mapped poses a practical barrier to the routine functional parcellation of individual human cortex. The present success in obtaining stimulus-driven maps in parietal cortex likely reflects the combined influence of several incremental improvements in the paradigm. Retinotopic mapping studies have long been plagued by subject difficulties in maintaining fixation and/or arousal level, because of the monotonous nature of the stimuli. To improve subject fixation and vigilance, a simple foveal dimming detection paradigm was used. Although endogenously directing attention to the foveal task may reduce the amount of exogenous attention allocated to the flashing checkerboard stimuli (Lavie, 1995; Rees et al., 1997; Schwartz et al., 2005), the benefits in terms of stable fixation and vigilance outweigh this reduction. Additional factors potentially aiding the detection of parietal maps include technical considerations (e.g., use of a surface coil) as well as aspects of the stimulus itself (e.g., chromatic contrast, continuous motion, long duty cycle of angular stimulation).

The anatomic position and topography of the IPS1/2 maps we find here are in excellent agreement with the saccade and attention maps of Schluppeck et al. (2005) and Silver et al. (2005). However, these authors note a discrepancy between the Talairach positions of the IPS1/2 maps and the parietal hemifield map found by Sereno et al. (2001), suggesting that these may be distinct areas. Here, we have used the same software for atlas alignment and registration as Sereno et al. (2001) and find relatively good agreement between the coordinates of our IPS1/2 and those of Silver et al. (2005) and Schluppeck et al. (2005) (Table 2). The parietal saccade map of Sereno et al. (2001) is in the vicinity of our IPS2/3/4; this map was described as "often" having an upper field representation positioned superiorly, consistent with the topography of IPS1 and IPS3. More recently, Sereno and Huang (2006) have reported a superior parietal map with aligned visual and tactile receptive fields responsive to stimuli on or near the face, located in anterior IPS at the level of the postcentral sulcus. The reported coordinates of this map place it $\sim 15 \mathrm{~mm}$ anterior and lateral to IPS4. Kitada et al. (2006) have reported a multisensory region of anterior IPS responsive during both visual and tactile orientation judgments, which also appears to be anterior and lateral to IPS4. These authors found extensive parietal activation, including activation of posterior parietal cortex, when contrasting tactile sensorimotor and motor tasks similar to the tactile tasks used here.

\section{Potential homologies and nomenclature}

Whereas caution is needed when evaluating possible homologies between human and nonhuman primates (Orban et al., 2004), there is increasing evidence to support an approximate homology between one or more of the V7 and IPS1/2 hemifield maps and macaque area LIP or its subdivisions (Lewis and Van Essen, 2000). Homology can be evaluated on several criteria, including functional properties, anatomical location, and topography. IPS1/2 and cells in macaque LIP show similar functional properties, including delay period responses and oculocentric spatial updating during delayed saccade tasks (Gnadt and Andersen, 1988; Duhamel et al., 1992; Medendorp et al., 2003; Merriam et al., 2003; Koyama et al., 2004; Schluppeck et al., 2006). Although 
the topography of macaque LIP is weaker than in earlier visual areas, a central visual field representation is consistently found dorsally along the lateral bank of the sulcus, whereas peripheral receptive fields are located ventrally (Blatt et al., 1990; Ben Hamed et al., 2001). Human V7 and IPS1/2 are organized similarly to macaque LIP in the eccentricity dimension, with a lateral central field representation and medial peripheral representation. However, the central field representation in humans is found near to the fundus of the sulcus, whereas the peripheral representation is located on the medial bank, consistent with a human LIP homolog shifting from the lateral to the medial sulcal bank (Grefkes and Fink, 2005) while retaining the same topographic orientation.

Potential homologies with established cortical areas in nonhuman primates may suggest that similar nomenclature be used
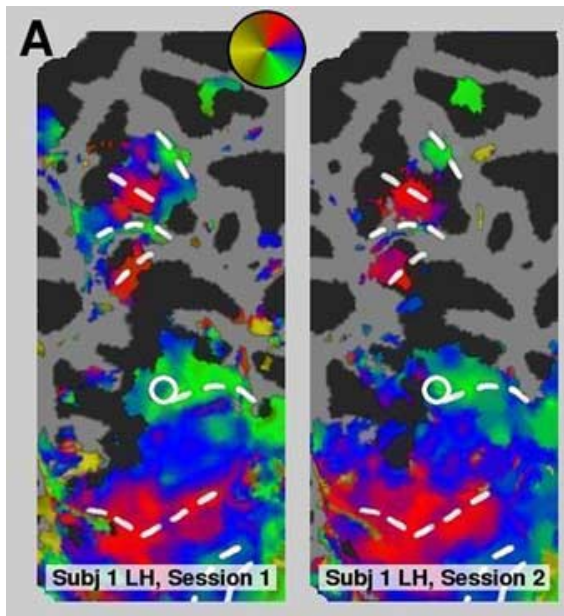

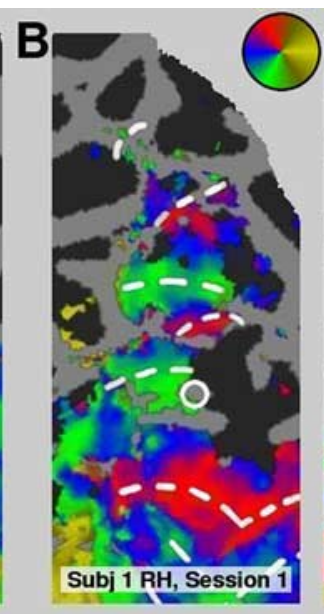

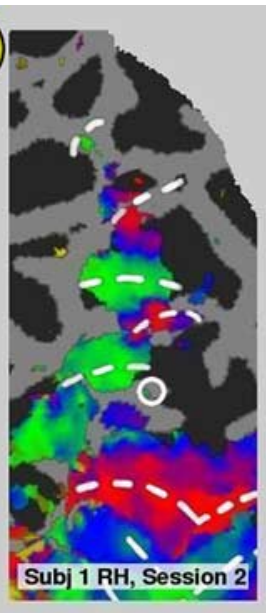

Figure 6. Test-retest reliability of visual field maps. Maps acquired during separate sessions of stimulus-driven mapping (at least 2 months apart) are shown projected onto parietal patches for both hemispheres of a single representative subject. The maps show qualitatively good reproducibility across sessions. In addition to the reproducibility of the maps themselves, large dropouts in the visuotopic response [as seen for instance at the level of IPS1 in the left hemisphere of subject 1 (subj 1)] are also reliably observed in the same positions across sessions. $\boldsymbol{A}$, Left hemisphere (LH). B, Right hemisphere (RH).

in humans. But, as many others have noted

(Orban et al., 2004; Sereno and Tootell, 2005; Wandell et al., 2005), it is quite likely that there exist areas in human cortex with no precise monkey homolog, limiting the extensibility of such a naming scheme. In suggesting the names IPS3 and IPS4 for the new hemifield visual maps, we have chosen to follow the naming convention established by Silver et al. (2005) and Schluppeck et al. (2005), because the IPS3/4 maps often appear as a continuation of this same visuotopic complex.

Tootell et al. (1998) provisionally defined V7 as a quadrant representation adjacent and anterior to area V3A. Subsequent investigations (Press et al., 2001; Tyler et al., 2005) showed that this area contains a full hemifield map and a distinct foveal representation. We note that V7 (rather than IPS1) is now seen to be the first (posteriormost) hemifield map on the medial bank of posterior IPS. A comparison of Talairach coordinates reported in previous studies (Table 3 ) indicates that regions previously referred to as inferior or posterior IPS (Wojciulik and Kanwisher, 1999; Simon et al., 2002; Xu and Chun, 2006) correspond to V7. This observation is significant because previous studies, in some cases, have incorrectly assumed that posterior/inferior IPS was not activated by direct stimulation. Thus, the present results may force a reconsideration of the interpretation of some previous visual attention and visual short-term memory results. Furthermore, the foveal representation of $\mathrm{V} 7$ is confluent with that of IPS1, suggesting the two maps form a unitary complex or cluster (Balasubramanian et al., 2002; Wandell et al., 2005). The existing nomenclature misleadingly implies the two maps are markedly anatomically distinct. A minimally disruptive change that may help resolve this taxonomic confusion would be to refer to V7 as "IPS0," better reflecting its anatomical location.

\section{Individual differences and tactile activation}

Although a consistent pattern of IPS maps is evident across the population of subjects, the precise topography of these maps varies from individual to individual. In many, but not all, subjects, the anterior IPS3/4 maps are rotated mediolaterally relative to the posterior IPS1/2 maps, and many hemispheres show substantial gaps or dropouts in the map complex. The presence of these dropouts is consistent with previous reports (Schluppeck et al., 2005; Silver et al., 2005), but their size and prevalence calls into

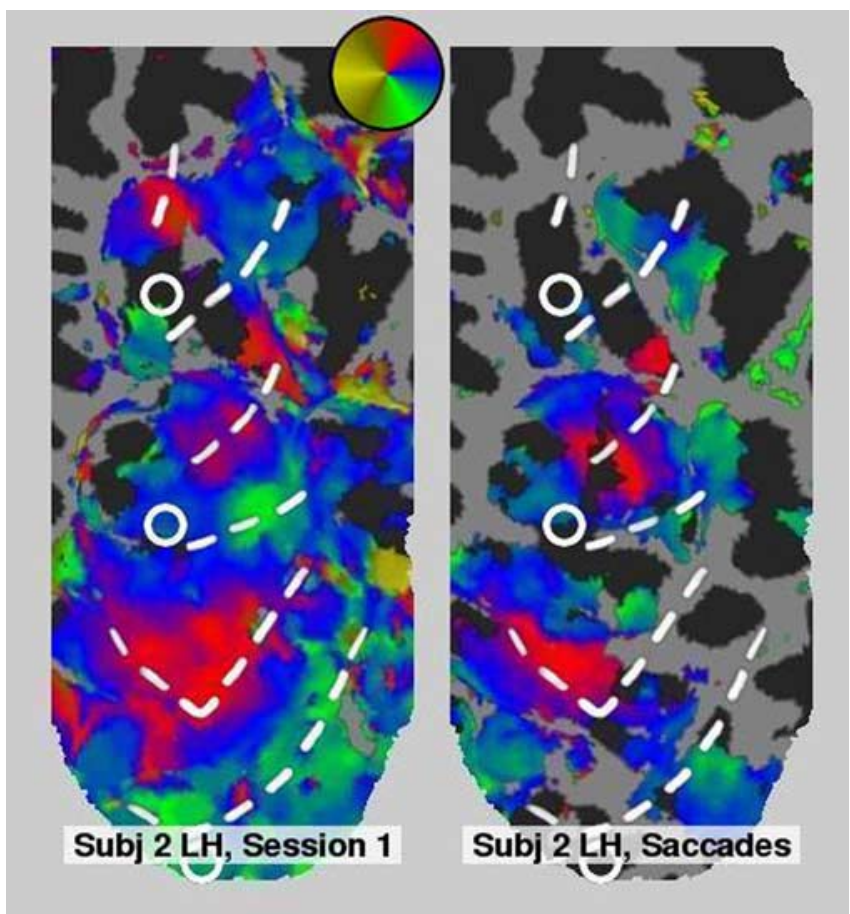

Figure 7. Comparison of stimulus-driven and saccade maps. Both stimulus-driven and saccade maps acquired in separate sessions for a hemisphere with relatively extensive saccade responses are shown. Each stimulus type produces similar angular phase maps, although we typically find a larger region of significant response during stimulus-driven mapping. Subj 2 , Subject 2; LH, left hemisphere.

question the characterization of this region as typically comprising a single, contiguous band of visual maps. This variability in the patterns of visuotopic response is reflected in complementary patterns of activation observed during a simple tactile task. The consistent appearance of tactile activation within the visuotopic dropouts provides additional evidence that these gaps in the map complex are truly present in the underlying cortical organization and do not result simply from methodological limitations, such as insufficient signal averaging. Additionally, that these gaps are 


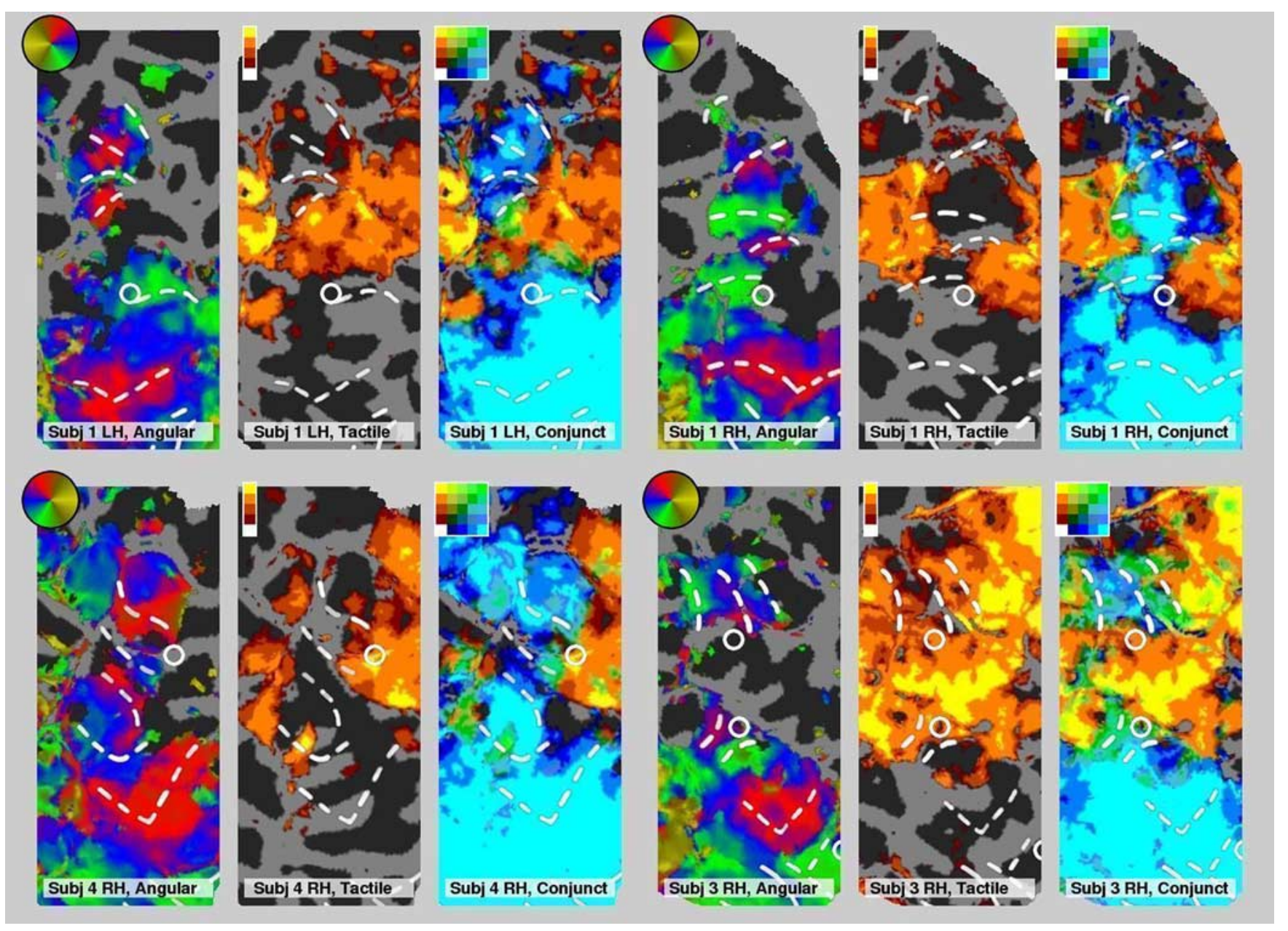

Figure 8. Visuotopic and tactile activations form complementary patterns in posterior parietal cortex. Four hemispheres from three subjects are shown, each with visuotopic maps, tactile activations, and the conjunction (Conjunct) of visuotopic and tactile activations overlaid. Tactile patches show activation in the contrast of roughness judgment versus a motor control. The discrete heat scale for the tactile map indicates significances at levels of $p<0.1,0.01,10^{-5}$, and $10^{-20}$ in progressively warmer colors. In the conjunction maps, significant tactile activity is indicated in warm colors along the vertical axis using the same scale as in the tactile maps, whereas significant visuotopic activity is shown in cool colors along the horizontal axis at levels of $p<0.1,0.05,0.01$, and $10^{-5}$. The conjunction of significant activation in both tasks (logical "and") is shown in shades of green. The minimum threshold for both tasks is set at a lenient 0.1 level, because the logical and conjunction is intrinsically a conservative statistic (Nichols et al., 2005). The activation patterns overlap at the edges; however, in no case have we observed tactile activation fully overlapping a connected region of visuotopic activation (or vice versa). Notably, in hemispheres where the visuotopic activation pattern is separated into two noncontiguous clusters of significant activity, the tactile activation "fills in" this gap (Subj 1 LH, Subj 3 RH). Conversely, in hemispheres with a continuous band of significant visuotopic activity, the tactile activation pattern is divided by this band into distinct, noncontiguous medial and lateral foci (Subj 1 RH, Subj 4 RH). Subj, Subject; LH, left hemisphere; RH, Right hemisphere.

Table 2. Distance of map centers from previously reported coordinates (in millimeters)

\begin{tabular}{lllllllllll}
\hline & Ser01 LIP & Sim02 pIPS & Sil05 V7 & Sil05 IPS1 & Sil05 IPS2 & Sch05 V7 & Sch05 IPS1 & Sch05 IPS2 & Ser06 VIP & Kit06 IPS \\
\hline V7 & 26 & $\mathbf{3}$ & $\mathbf{9}$ & $\mathbf{7}$ & 17 & $\mathbf{7}$ & $\mathbf{1 1}$ & 21 & 46 & 40 \\
IPS1 & 15 & $\mathbf{1 0}$ & 20 & $\mathbf{8}$ & $\mathbf{1 1}$ & 18 & $\mathbf{8}$ & $\mathbf{1 2}$ & 36 \\
IPS2 & $\mathbf{4}$ & 23 & 32 & 18 & 13 & 30 & 16 & $\mathbf{9}$ & 27 \\
IPS3 & $\mathbf{5}$ & 28 & 38 & 24 & 20 & 36 & 23 & 16 & 22 \\
IPS4 & $\mathbf{6}$ & 30 & 40 & 27 & 24 & 38 & 26 & 20 & 19 \\
\hline
\end{tabular}

Ser01, Sereno et al.,2001; Sim02, Simon et al., 2002 (pointing): Sil05, Silver et al., 2005; Sch05, Schluppeck et al., 2005; Ser06, Sereno and Huang, 2006; Kit06, Kitada et al., 2006. Where these previous studies specified positions relative to the Talairach atlas, we converted our MNI (Montreal Neurological Institute) coordinates into Talairach space via the algorithm implemented in Brett's mni2tal before calculating the distance between points. Bold indicates three-dimensional distances $<1$ SD.

present in some but not all hemispheres, even within the same subject (Fig. 8, compare $A, B$ ), indicates substantial variability in the functional organization of parietal cortex, possibly reflecting either differing sensory experiences during development or continuing neuroplasticity into adulthood.

Despite the clear dissociation between visuotopic and tactile responses, these patterns do not necessarily indicate the presence of two or more functionally distinct, unimodal regions. Alternatively, the two tasks may drive separate portions of the same large-scale multisensory spatial map. During the tactile task, the stimulated right index finger was placed by the subject's side as they lay supine in the scanner, putting it outside the field of vision even if the blindfold were to be removed. Thus, the primarily disjoint tactile and visuotopic activations may be attributable to the distinct regions of space occupied by the two stimuli in some nonretinotopic underlying coordinate system (Snyder et al., 1998; Colby and Goldberg, 1999; Cohen and Andersen, 2002; Avillac et al., 2005). The presence or absence of apparent gaps in 
Table 3. Distance of map centers from previously reported coordinates for attention and visual short-term memory activations (in millimeters)

\begin{tabular}{|c|c|c|c|c|c|c|c|c|}
\hline & Cul98 antIPS & Kas99 SPL & Woj99 IPS & Sim02 SPL & Tod04 IPS & Srcs05 IPS & Xu06 InfIPS & Xu06 SupIPS \\
\hline V7 & 22 & 29 & 12 & 33 & 18 & 15 & 9 & 20 \\
\hline IPS1 & 11 & 18 & 6 & 21 & 7 & 6 & 19 & 10 \\
\hline IPS2 & 3 & 6 & 15 & 9 & 8 & 11 & 31 & 8 \\
\hline IPS3 & 8 & 10 & 18 & 6 & 11 & 17 & 37 & 10 \\
\hline IPS4 & 12 & 13 & 20 & 10 & 13 & 19 & 39 & 11 \\
\hline
\end{tabular}

Cul98, Culham et al., 1998; Kas99, Kastner et al., 1999; Wo99, Wojciulik et al., 1999; Sim02, Simon et al., 2002 (attention); Tod04, Todd and Marois, 2004; Srcs05, Serences et al., 2005; Xu06, Xu and Chun, 2006; antIPS, anterior IPS; SPL, superior parietal lobule; InfIPS, inferior IPS; SupIPS, superior IPS. Bold indicates three-dimensional distances $<1$ SD.

the visuotopic complex may then result from intersubject variability in the scaling of this spatial representation (Dougherty et al., 2003; Duncan and Boynton, 2003), with the space outside the visual field represented to varying degrees in different individuals, rather than reflecting intersubject differences in the adjacency relationships of functionally distinct cortical areas. Determining the precise coordinate system in use in these posterior parietal areas remains an interesting topic for future research.

\section{Functional significance of IPS0/1/2/3/4}

Previous functional imaging studies have demonstrated that numerous functions, including saccades (Sereno et al., 2001; Simon et al., 2002; Astafiev et al., 2003; Schluppeck et al., 2005), multisensory integration (Kitada et al., 2006; Merabet et al., 2007), visual short-term memory (Todd and Marois, 2004; Xu and Chun, 2006), and visual attention (Culham et al., 1998; Kastner et al., 1999; Wojciulik and Kanwisher, 1999; McMains and Somers, 2004, 2005; Serences et al., 2005; Silver et al., 2005), strongly activate posterior parietal cortical circuitry. In many cases, Talairach coordinates reported in previous studies lie within 1 SD of one or more of the IPS regions identified here (Tables 2, 3). Part of the confusion in dissecting the functional roles of IPS may be attributed to the fact that many previous studies divided IPS into only two subregions, such as posterior and anterior. Here, five distinct cortical maps are demonstrated to exist in just a posterior subregion of the medial bank of IPS. As observed in the macaque (Lewis and Van Essen, 2000), these visually driven areas are much smaller than early visual cortical areas V1 and V2. Based on the small size of these visual regions, it is conjectured that human posterior parietal cortex may be quite dense with distinct cortical areas. The substantial individual differences reported here emphasize the utility of within-subject analysis of this functional circuitry. Reliable identification of these areas should provide a useful tool for the dissection of the sensory and cognitive processes occurring within this region of the human brain.

\section{References}

Astafiev SV, Shulman GL, Stanley CM, Snyder AZ, Van Essen DC, Corbetta M (2003) Functional organization of human intraparietal and frontal cortex for attending, looking, and pointing. J Neurosci 23:4689-4699.

Avillac M, Deneve S, Olivier E, Pouget A, Duhamel JR (2005) Reference frames for representing visual and tactile locations in parietal cortex. Nat Neurosci 8:941-949.

Balasubramanian M, Polimeni J, Schwartz EL (2002) The V1-V2-V3 complex: quasiconformal dipole maps in primate striate and extra-striate cortex. Neural Netw 15:1157-1163.

Behrmann M, Geng JJ, Shomstein S (2004) Parietal cortex and attention. Curr Opin Neurobiol 14:212-217.

Ben Hamed S, Duhamel JR, Bremmer F, Graf W (2001) Representation of the visual field in the lateral intraparietal area of macaque monkeys: a quantitative receptive field analysis. Exp Brain Res 140:127-144.

Blatt GJ, Andersen RA, Stoner GR (1990) Visual receptive field organization and cortico-cortical connections of the lateral intraparietal area (area LIP) in the macaque. J Comp Neurol 299:421-445.

Buneo CA, Andersen RA (2006) The posterior parietal cortex: sensorimotor interface for the planning and online control of visually guided movements. Neuropsychologia 44:2594-2606.

Cohen YE, Andersen RA (2002) A common reference frame for movement plans in the posterior parietal cortex. Nat Rev Neurosci 3:553-562.

Colby CL, Goldberg ME (1999) Space and attention in parietal cortex. Annu Rev Neurosci 22:319-349.

Connor CE, Hsiao SS, Phillips JR, Johnson KO (1990) Tactile roughness: neural codes that account for psychophysical magnitude estimates. J Neurosci 10:3823-3836.

Cox RW, Hyde JS (1997) Software tools for analysis and visualization of fMRI data. NMR Biomed 10:171-178.

Culham JC, Kanwisher NG (2001) Neuroimaging of cognitive functions in human parietal cortex. Curr Opin Neurobiol 11:157-163.

Culham JC, Brandt SA, Cavanagh P, Kanwisher NG, Dale AM, Tootell RB (1998) Cortical fMRI activation produced by attentive tracking of moving targets. J Neurophysiol 80:2657-2670.

Dale AM, Fischl B, Sereno MI (1999) Cortical surface-based analysis. I. Segmentation and surface reconstruction. NeuroImage 9:179-194.

DeYoe EA, Carman GJ, Bandettini P, Glickman S, Wieser J, Cox R, Miller D, Neitz J (1996) Mapping striate and extrastriate visual areas in human cerebral cortex. Proc Natl Acad Sci USA 93:2382-2386.

Dougherty RF, Koch VM, Brewer AA, Fischer B, Modersitzki J, Wandell BA (2003) Visual field representations and locations of visual areas $V 1 / 2 / 3$ in human visual cortex. J Vis 3:586-598.

Duhamel JR, Colby CL, Goldberg ME (1992) The updating of the representation of visual space in parietal cortex by intended eye movements. Science 255:90-92.

Duncan RO, Boynton GM (2003) Cortical magnification within human primary visual cortex correlates with acuity thresholds. Neuron 38:659-671.

Engel SA, Rumelhart DE, Wandell BA, Lee AT, Glover GH, Chichilnisky EJ, Shadlen MN (1994) fMRI of human visual cortex. Nature 369:525.

Fischl B, Sereno MI, Dale AM (1999) Cortical surface-based analysis. II: Inflation, flattening, and a surface-based coordinate system. NeuroImage 9:195-207.

Fisher NI, Lee AJ (1983) A correlation-coefficient for circular data. Biometrika 70:327-332.

Gnadt JW, Andersen RA (1988) Memory related motor planning activity in posterior parietal cortex of macaque. Exp Brain Res 70:216-220.

Grefkes C, Fink GR (2005) The functional organization of the intraparietal sulcus in humans and monkeys. J Anat 207:3-17.

Haxby JV, Gobbini MI, Furey ML, Ishai A, Schouten JL, Pietrini P (2001) Distributed and overlapping representations of faces and objects in ventral temporal cortex. Science 293:2425-2430.

Horton JC, Hoyt WF (1991) The representation of the visual field in human striate cortex. A revision of the classic Holmes map. Arch Ophthalmol 109:816-824.

Hubbard EM, Piazza M, Pinel P, Dehaene S (2005) Interactions between number and space in parietal cortex. Nat Rev Neurosci 6:435-448.

Kastner S, Ungerleider LG (2000) Mechanisms of visual attention in the human cortex. Annu Rev Neurosci 23:315-341.

Kastner S, Pinsk MA, De Weerd P, Desimone R, Ungerleider LG (1999) Increased activity in human visual cortex during directed attention in the absence of visual stimulation. Neuron 22:751-761.

Kitada R, Hashimoto T, Kochiyama T, Kito T, Okada T, Matsumura M, Lederman SJ, Sadato N (2005) Tactile estimation of the roughness of gratings yields a graded response in the human brain: an fMRI study. NeuroImage 25:90-100.

Kitada R, Kito T, Saito DN, Kochiyama T, Matsumura M, Sadato N, Lederman SJ (2006) Multisensory activation of the intraparietal area when 
classifying grating orientation: a functional magnetic resonance imaging study. J Neurosci 26:7491-7501.

Koyama M, Hasegawa I, Osada T, Adachi Y, Nakahara K, Miyashita Y (2004) Functional magnetic resonance imaging of macaque monkeys performing visually guided saccade tasks: comparison of cortical eye fields with humans. Neuron 41:795-807.

Larsson J, Landy MS, Heeger DJ (2006) Orientation-selective adaptation to first- and second-order patterns in human visual cortex. J Neurophysiol 95:862-881.

Lavie N (1995) Perceptual load as a necessary condition for selective attention. J Exp Psychol Hum Percept Perform 21:451-468.

Lewis JW, Van Essen DC (2000) Mapping of architectonic subdivisions in the macaque monkey, with emphasis on parieto-occipital cortex. J Comp Neurol 428:79-111.

McMains SA, Somers DC (2004) Multiple spotlights of attentional selection in human visual cortex. Neuron 42:677-686.

McMains SA, Somers DC (2005) Processing efficiency of divided spatial attention mechanisms in human visual cortex. J Neurosci 25:9444-9448.

Medendorp WP, Goltz HC, Vilis T, Crawford JD (2003) Gaze-centered updating of visual space in human parietal cortex. J Neurosci 23:6209-6214.

Merabet L, Thut G, Murray B, Andrews J, Hsiao S, Pascual-Leone A (2004) Feeling by sight or seeing by touch? Neuron 42:173-179.

Merabet LB, Swisher JD, McMains SA, Halko MA, Amedi A, Pascual-Leone A, Somers DC (2007) Combined activation and deactivation of visual cortex during tactile sensory processing. J Neurophysiol 97:1633-1641.

Merriam EP, Colby CL (2005) Active vision in parietal and extrastriate cortex. Neuroscientist 11:484-493.

Merriam EP, Genovese CR, Colby CL (2003) Spatial updating in human parietal cortex. Neuron 39:361-373.

Mesulam MM, Small DM, Vandenberg R, Gitelman DR, Nobre AC (2005) A heteromodal large-scale network for spatial attention. In: Neurobiology of attention (Itti L, Rees G, Tsotos J, eds), pp 29-34. San Diego: Elsevier.

Mitra PP, Pesaran B (1999) Analysis of dynamic brain imaging data. Biophys J 76:691-708.

Nichols T, Brett M, Andersson J, Wager T, Poline JB (2005) Valid conjunction inference with the minimum statistic. NeuroImage 25:653-660.

Ono M, Kubik S, Abernathey CD (1990) Atlas of the cerebral sulci. Stuttgart: Georg Thieme Verlag.

Orban GA, Van Essen D, Vanduffel W (2004) Comparative mapping of higher visual areas in monkeys and humans. Trends Cogn Sci 8:315-324.

Orban GA, Claeys K, Nelissen K, Smans R, Sunaert S, Todd JT, Wardak C, Durand JB, Vanduffel W (2006) Mapping the parietal cortex of human and non-human primates. Neuropsychologia 44:2647-2667.

Percival DB, Walden AT (1993) Spectral analysis for physical applications: multitaper and conventional univariate techniques. Cambridge, UK: Cambridge UP.

Press WA, Brewer AA, Dougherty RF, Wade AR, Wandell BA (2001) Visual areas and spatial summation in human visual cortex. Vision Res 41:1321-1332.

Rees G, Frith CD, Lavie N (1997) Modulating irrelevant motion perception by varying attentional load in an unrelated task. Science 278:1616-1619.

Saxe R, Brett M, Kanwisher N (2006) Divide and conquer: a defense of functional localizers. NeuroImage 30:1088-1096; discussion 1097-1089.

Schluppeck D, Glimcher P, Heeger DJ (2005) Topographic organization for delayed saccades in human posterior parietal cortex. J Neurophysiol 94:1372-1384.

Schluppeck D, Curtis CE, Glimcher PW, Heeger DJ (2006) Sustained activity in topographic areas of human posterior parietal cortex during memory-guided saccades. J Neurosci 26:5098-5108.

Schneider KA, Richter MC, Kastner S (2004) Retinotopic organization and functional subdivisions of the human lateral geniculate nucleus: a highresolution functional magnetic resonance imaging study. J Neurosci 24:8975-8985.

Schwartz S, Vuilleumier P, Hutton C, Maravita A, Dolan RJ, Driver J (2005) Attentional load and sensory competition in human vision: modulation of fMRI responses by load at fixation during task-irrelevant stimulation in the peripheral visual field. Cereb Cortex 15:770-786.
Serences JT, Shomstein S, Leber AB, Golay X, Egeth HE, Yantis S (2005) Coordination of voluntary and stimulus-driven attentional control in human cortex. Psychol Sci 16:114-122.

Sereno MI, Huang RS (2006) A human parietal face area contains aligned head-centered visual and tactile maps. Nat Neurosci 9:1337-1343.

Sereno MI, Tootell RB (2005) From monkeys to humans: what do we now know about brain homologies? Curr Opin Neurobiol 15:135-144.

Sereno MI, Dale AM, Reppas JB, Kwong KK, Belliveau JW, Brady TJ, Rosen BR, Tootell RB (1995) Borders of multiple visual areas in humans revealed by functional magnetic resonance imaging. Science 268:889-893.

Sereno MI, Pitzalis S, Martinez A (2001) Mapping of contralateral space in retinotopic coordinates by a parietal cortical area in humans. Science 294:1350-1354.

Shmuel A, Yacoub E, Pfeuffer J, Van de Moortele PF, Adriany G, Hu X, Ugurbil K (2002) Sustained negative BOLD, blood flow and oxygen consumption response and its coupling to the positive response in the human brain. Neuron 36:1195-1210.

Silver MA, Ress D, Heeger DJ (2005) Topographic maps of visual spatial attention in human parietal cortex. J Neurophysiol 94:1358-1371.

Simon O, Mangin JF, Cohen L, Le Bihan D, Dehaene S (2002) Topographical layout of hand, eye, calculation, and language-related areas in the human parietal lobe. Neuron 33:475-487.

Slepian D (1978) Prolate spheroidal wave functions, Fourier analysis, and uncertainty. V. The discrete case. Bell Syst Tech J 57:1371-1430.

Smith AT, Greenlee MW, Singh KD, Kraemer FM, Hennig J (1998) The processing of first- and second-order motion in human visual cortex assessed by functional magnetic resonance imaging (fMRI). J Neurosci 18:3816-3830.

Snyder LH, Grieve KL, Brotchie P, Andersen RA (1998) Separate body- and world-referenced representations of visual space in parietal cortex. Nature 394:887-891.

Swisher J, Crum K, McMains S, Halko M, Sheremata S, Somers DC (2005) Stimulus-driven retinotopic maps in human parietal cortex observed via fMRI. Soc Neurosci Abstr 31:582.12.

Thomson DJ (1982) Spectrum estimation and harmonic analysis. Proc IEEE 70:1055-1096.

Todd JJ, Marois R (2004) Capacity limit of visual short-term memory in human posterior parietal cortex. Nature 428:751-754.

Tootell RB, Mendola JD, Hadjikhani NK, Ledden PJ, Liu AK, Reppas JB, Sereno MI, Dale AM (1997) Functional analysis of V3A and related areas in human visual cortex. J Neurosci 17:7060-7078.

Tootell RB, Hadjikhani N, Hall EK, Marrett S, Vanduffel W, Vaughan JT, Dale AM (1998) The retinotopy of visual spatial attention. Neuron 21:1409-1422.

Tyler CW, Likova LT, Chen CC, Kontsevich LL, Schira MM, Wade AR (2005) Extended concepts of occipital retinotopy. Curr Med Imaging Rev 1:319-329.

Van Essen DC (2004) Organization of visual areas in macaque and human cerebral cortex. In: The visual neurosciences (Chalupa LM, Werner JS, eds), pp 507-521. Cambridge, MA: MIT.

Wagner AD, Shannon BJ, Kahn I, Buckner RL (2005) Parietal lobe contributions to episodic memory retrieval. Trends Cogn Sci 9:445-453.

Wandell BA, Brewer AA, Dougherty RF (2005) Visual field map clusters in human cortex. Philos Trans R Soc Lond B Biol Sci 360:693-707.

Warnking J, Dojat M, Guerin-Dugue A, Delon-Martin C, Olympieff S, Richard N, Chehikian A, Segebarth C (2002) fMRI retinotopic mappingstep by step. NeuroImage 17:1665-1683.

Wojciulik E, Kanwisher N (1999) The generality of parietal involvement in visual attention. Neuron 23:747-764.

Xu Y, Chun MM (2006) Dissociable neural mechanisms supporting visual short-term memory for objects. Nature 440:91-95.

Yantis S, Serences JT (2003) Cortical mechanisms of space-based and object-based attentional control. Curr Opin Neurobiol 13:187-193.

Zarahn E, Aguirre GK, D’Esposito M (1997) Empirical analyses of BOLD fMRI statistics. I. Spatially unsmoothed data collected under nullhypothesis conditions. NeuroImage 5:179-197. 